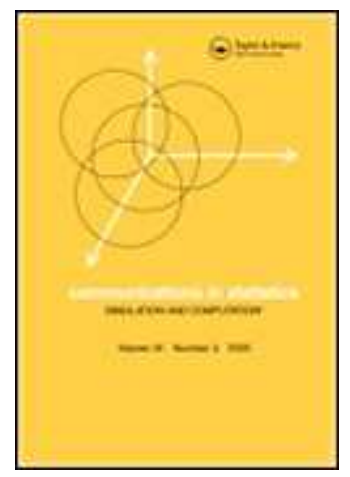

\title{
Confidence intervals for reliability of stress-strength models in the normal case
}

\begin{tabular}{|c|c|}
\hline Journal: & Communications in Statistics - Simulation and Computation \\
\hline Manuscript ID: & LSSP-2010-0183.R2 \\
\hline Manuscript Type: & Original Paper \\
\hline $\begin{array}{r}\text { Date Submitted by the } \\
\text { Author: }\end{array}$ & 28-Jan-2011 \\
\hline Complete List of Authors: & $\begin{array}{l}\text { Barbiero, Alessandro; Università degli Studi di Milano, Department } \\
\text { of Economics, Business and Statistics }\end{array}$ \\
\hline Keywords: & $\begin{array}{l}\text { asymptotically normal, independent normal, parametric bootstrap, } \\
\text { Monte Carlo simulations, stress-strength, variance estimation }\end{array}$ \\
\hline Abstract: & $\begin{array}{l}\text { In this paper we propose some procedures to get confidence } \\
\text { intervals for the reliability in stress-strength models. The } \\
\text { confidence intervals are obtained either through a parametric } \\
\text { bootstrap procedure or using asymptotic results, and are applied to } \\
\text { the particular context of two independent normal random variables. } \\
\text { The performance of these estimators and other known approximate } \\
\text { estimators are empirically checked through a simulation study } \\
\text { which considers several scenarios. }\end{array}$ \\
\hline \multicolumn{2}{|c|}{$\begin{array}{l}\text { Note: The following files were submitted by the author for peer review, but cannot be converted } \\
\text { to PDF. You must view these files (e.g. movies) online. }\end{array}$} \\
\hline reliability_second_revision.te & \\
\hline
\end{tabular}

\section{SCHOLARONE ${ }^{\text {M }}$ Manuscripts}




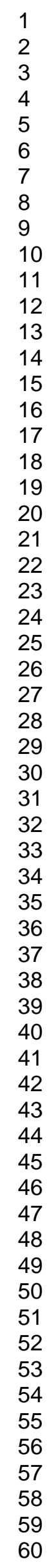




\section{Reply to Editor and Reviewers' comments on manuscript "Confidence intervals for reliability of stress-strength models in the normal case" by A. Barbiero}

I would like to thank the Editor, Associate Editor and two Referees for their very helpful comments on my submitted manuscript. I agree with all their comments and believe that they will contribute to the improvement of the paper.

You will find the modifications/additions to the original text coloured in red in the pdf file I have enclosed ( $\backslash$ textcolor $\{r e d\}\{\}$ in the LaTeX file). I have corrected some minor typos or imprecisions (e.g. added bold font for vectors of parameters in some formulas).

A point-to-point list of responses is given below (my answers after "R:").

\section{Reviewer \#1}

Comments to the Author

Comments on the paper "Confidence intervals for reliability in the normal case" by Barbiero, Alessandro

This paper considers a bootstrap and some approximate methods for the interval estimation of reliability for normally distributed

stress and strength. The paper is nicely written and can be easily followed.

R: Thanks for your appreciation.

\section{Reviewer \#2}

Comments to the Author

Comments on the paper "Confidence Intervals for Reliability in the Normal Case" by Barbiero The revised version looks better than the first submission, but still I have some concerns.

1. The title should include the term "stress-strength", not just reliability.

$\mathrm{R}$ : Indeed "reliability" alone is a too vague term; I have changed the title, which now is "Confidence intervals for reliability of stress-strength models in the normal case"

2. Page 4, below Eqn (2): Write "Chang's method is overly conservative producing CIs that are unnecessarily wider."

R: Done 
3. Page 5, line 10 from below: noncentral t, not noncentral Student's T.

R: Done
4. Page 5: Cumulative distribution function, not cumulative density function.
R: Done

5. Section 3, first line: We will now describe our new method....

R: Done
6. Section 3.1: Check the reference to Efron's book.
$\mathrm{R}$ : checked; now it is referenced to as "Efron and Tibshirani, 1994"

7. I also have some concerns with the description of the PB approach. Note that all procedures depend on only the sample means and variances, and so we do not have to generate sample from $\$ \mathrm{~N}\left(\operatorname{lbar}\{\mathrm{x}\} ; \mathrm{s}^{\wedge} 2 \_\mathrm{x}\right) \$$ and $\$ \mathrm{~N}\left(\operatorname{lbar}\{\mathrm{y}\} ; \mathrm{s}^{\wedge} 2 \_\mathrm{y}\right) \$$. Indeed, we only have to generate $\$ \operatorname{lbar}\{\mathrm{x}\}^{\wedge} * \mid \operatorname{sim}$ $\mathrm{N}\left(\operatorname{lbar}\{\mathrm{x}\} ; \mathrm{s}^{\wedge} 2 \_\mathrm{x} / \mathrm{n}\right) \$$ and $\$ \mathrm{~s}^{\wedge}\left\{2^{*}\right\} \_\mathrm{x} \backslash \operatorname{sim} \mathrm{s}^{\wedge} 2 \_\mathrm{x} \mid \mathrm{chi}{ }^{\wedge} 2 \_\{\mathrm{n}-1\}(\mathrm{n}-1) \$$. Similarly, $\$ \operatorname{bar}\{\mathrm{y}\}^{\wedge} * \$$ and $\$ \mathbf{s}^{\wedge}\{* 2\} \_\mathrm{y} \$$ can be generated.

$\mathrm{R}$ : due to the properties of (the sample mean of) the normal distribution, actually one can directly generate the sample means and variances, but in a more general case one has to bootstrap the original distribution. I have inserted your note in the algorithm: "... since all procedures depend on only the sample means and variances, for the well-known properties of the normal distribution, for this case we only have to generate $\$ \operatorname{lbar}\{X\}^{\wedge *} \mid \operatorname{sim} N\left(\operatorname{lbar}\{x\} ; s^{\wedge} 2 \_x / n\right) \$$ and $\$ S^{\wedge}\left\{2^{*}\right\} \_x \mid \operatorname{sim}$ $\mathrm{S}^{\wedge} 2 \_x \mid c h i \wedge 2 \_\{n-1\} /(n-1) \$$. Similarly, $\$ \backslash b a r\{Y\}^{\wedge * \$}$ and $\$ S^{\wedge}\{* 2\} \_y \$$ can be generated."

8. The asymptotic procedures perform well for constructing two-sided CIs, but not for one-sided CIs. Indeed, Krishnamoorthy and Lin (2010) noted that for the Weibull case asymptotic CIs overcover on one-tail and undercover on the other, and so overall coverage probabilities of asymptotic two-sided CIs seem to be OK. I observe similar behavior for the normal case also. The author should note this point.

R: Thanks for the explanation; I put a note when commenting the results for the one-side case: "One should also note an overall light worsening of the performance of asymptotic procedures passing from two-sided to one-sided CIs: this may be ascribed to the fact that asymptotic CIs overcover on one-tail and undercover on the other, and so overall coverage probabilities of asymptotic two-sided 
CIs turn out to be close to the nominal \$1-lalpha\$. This behavior has been detected by lcite $\{$ Kri $\}$ for the Weibull case." 


\title{
Confidence intervals for reliability of stress-strength models in the normal case
}

\author{
Alessandro Barbiero \\ Department of Economics, Business and Statistics, \\ Università degli Studi di Milano, Italy. \\ alessandro.barbiero@unimi.it
}

\begin{abstract}
In this paper we propose some procedures to get confidence intervals for the reliability in stress-strength models. The confidence intervals are obtained either through a parametric bootstrap procedure or using asymptotic results, and are applied to the particular context of two independent normal random variables. The performance of these estimators and other known approximate estimators are empirically checked through a simulation study which considers several scenarios.
\end{abstract}

Keywords: asymptotically normal, independent normal, parametric bootstrap, Monte Carlo simulations, stress-strength, variance estimation

\section{Introduction}

The aim of this paper is to propose confidence intervals for the probability $P(Y<X)$, where $X$ and $Y$ are independent random variables and when sample values $x$ and $y$ are observed. The recent years have seen a lot of publications on this subject: the basic impetus to these developments can be attributed to the specific practical problem of applied statistics summarized by 
the term "stress-strength". In the simplest terms this can be described as an assessment of "reliability" of a "component" in terms of random variables $Y$ representing "stress" experienced by the component and $X$ representing the "strength" of the component available to overcome the stress. According to this simplified scenario, if the stress exceeds the strength $(Y>X)$ the component would fail; and vice versa. Reliability is defined as the probability of not failing: $R=P(Y<X)$. The reliability problem arises in the fields of aeronautical, civil, mechanical and electronic engineering. For example, $X$ may be the breakdown voltage of a capacitor, while $Y$ may represent the voltage output of a transverter (Hall, 1984); or $X$ may be the chamber burst strength and $Y$ the operating pressure for a rocket-motor (Reiser and Guttman, 1986).

The beginning point of this idea was introduced by Birnbaum (1956) and developed by Birnbaum and McCarty (1958). The latter paper does for the first time include $P(Y<X)$ in its title. The formal term "stress-strength" appears for the first time in the title of the paper by Church and Harris (1970). In the course of time, there have been attempts to introduce further elements of adherence to reality, including various generalizations and applications; many papers are devoted both to merely probabilistic problems associated with the evaluation of $R$ and the construction of efficient and reliable estimators of this parameter, based on sample values with various assumptions on the distributions of $X$ and $Y$. So the research has not only dealt with the problems of deriving theoretical expressions for $P(Y<X)$ and its modifications and extensions under various distributional assumptions, but also with estimation of these probabilities based on samples of various structure, providing for approximations to variances and confidence bounds (Kotz et al., 2003). Many of these works presume that both random variables has distributions belonging to the same family (such as normal, exponential, log-normal, Weibull, etc.) and more importantly they assume independence between them. Some authors, however, considered the case in which $X$ and $Y$ admit a specified form of dependence (bivariate normal, bivariate exponential, etc.): see for example Mukherjee and Sharan (1985); Nadarajah and Kotz (2005); Roy (1993). Harris and Soms (1991) emphasize the fact that in many applications the "reliability" has to be very close to one for the device to have any possibility of "useful" life: this implies that very large samples may be needed to obtain sufficiently precise estimates of reliability, since we are dealing with extreme tails of distributions.

In this paper we will consider the estimation of reliability when $X$ and $Y$ are independent 
random variables and their distribution belongs to a known family (normal) with unknown parameters. Based upon ML estimators, we will consider and refine approximate interval estimators already appeared in literature, we will propose an original bootstrap interval estimator and empirically investigate and compare their performance in terms of coverage and accuracy through an extensive simulation study.

The paper is structured as follows: in Section 2 we describe available methods for interval estimation of reliability for an independent setup; in Section 3 we describe our proposals; in Section 4 we describe the simulation design, show the results and discuss them; in Section 5, we present a real application, in Section 6 we give some final remarks.

\section{Available methods}

We will now briefly introduce the concept of "reliability" for a stress-strength model and summarize the methods available in literature for its interval estimation. We will confine ourselves to the parametric case only.

Let the strength be modeled by r.v. $X$ and the stress by r.v. $Y$. Let us suppose that $X$ follows a continuous distribution with vector of parameters $\boldsymbol{\theta}$ and $Y$ follows a continuous distributions with vector of parameters $\boldsymbol{\lambda}$. The reliability of a stress-strength model is formally defined as

$$
R=P(Y<X)=P(Y-X<0)
$$

and the following expression can be used to obtain the reliability:

$$
R=\int_{-\infty}^{+\infty} f_{x}(x, \boldsymbol{\theta}) \int_{-\infty}^{x} f_{y}(y, \boldsymbol{\lambda}) d y d x
$$

where $f_{x}$ and $f_{y}$ denote the p.d.f. of $X$ and $Y$ respectively. For independent normal r.v. $X \sim$ $N\left(\mu_{x}, \sigma_{x}\right)$ and $Y \sim N\left(\mu_{y}, \sigma_{y}\right)$, the reliability presents the easy expression

$$
R=\Phi\left(\frac{\mu_{x}-\mu_{y}}{\sqrt{\sigma_{x}^{2}+\sigma_{y}^{2}}}\right)
$$

where $\Phi$ denotes the standard normal c.d.f.

A straightforward procedure for getting conservative confidence intervals for the reliability $R$ was described by Chang (Chang, 1995). The basic idea was to start from confidence intervals 
for the parameters of the two r.v. and then numerically obtain a lower and upper bound for reliability from the lower and upper bounds of these intervals. Chang's method provides for the reliability of a stress-strength model with two independent normal variables the following conservative $100 \cdot(1-2 \alpha)^{2} \%$ interval:

$$
[\underline{R}, \bar{R}]=\left[\inf _{\Omega} R, \sup _{\Omega} R\right]=\left[\Phi\left[\frac{\underline{\mu}_{x}-\bar{\mu}_{y}}{\sqrt{\bar{\sigma}_{x}^{2}+\bar{\sigma}_{y}^{2}}}\right], \Phi\left[\frac{\bar{\mu}_{x}-\underline{\mu}_{y}}{\sqrt{\underline{\sigma}_{x}^{2}+\underline{\sigma}_{y}^{2}}}\right]\right]
$$

where $\Omega=\left\{\underline{\mu_{x}} \leq \mu_{x} \leq \overline{\mu_{x}}, \underline{\mu_{y}} \leq \mu_{y} \leq \overline{\mu_{y}}, \underline{\sigma_{x}^{2}} \leq \sigma_{x}^{2} \leq \overline{\sigma_{x}^{2}}, \underline{\sigma_{y}^{2}} \leq \sigma_{y}^{2} \leq \overline{\sigma_{y}^{2}}\right\}$ and $\left(\underline{\mu_{x}}, \overline{\mu_{x}}\right)$ and $\left(\underline{\sigma_{x}^{2}}, \overline{\sigma_{x}^{2}}\right)$ are a $(1-\alpha)$ confidence interval for $\mu_{x}$ and $\sigma_{x}^{2}$ respectively, and analogously for the $Y$ parameters. Yet, Chang's method is overly conservative producing CIs that are unnecessarily wider.

Reiser and Guttman (1986) examine statistical inference for $P(Y<X)$, where $X$ and $Y$ are independent normal variates with unknown means and variances. Two approximate methods for obtaining confidence intervals and an approximate Bayesian probability interval are described. The actual coverage probabilities of these intervals are examined by simulation. Specifically, they first consider

$$
\delta=\frac{\mu_{x}-\mu_{y}}{\sqrt{\sigma_{x}^{2}+\sigma_{y}^{2}}}
$$

Since $R$ is a monotonic function of $\delta$, finding a CI for $R$ is equivalent to finding a CI for $\delta$. They argue that it seems more reasonable to base inference on a normal approximation to $\delta$ than on the normal approximation to $R$ since $\delta$ is unbounded, while $R$ is bounded in $(-1,1)$. An approximate $(1-\alpha)$ CI they propose is given by

$$
\left(\Phi\left(\delta_{L}\right), \Phi\left(\delta_{U}\right)\right)
$$

where $\delta_{L}$ and $\delta_{U}$ are the lower and upper bound of the interval

$$
\hat{\delta} \pm\left(\frac{1}{\hat{M}}+\frac{\hat{\delta}^{2}}{2 \hat{f}}\right) z_{1-\alpha / 2}
$$

with

$$
\begin{gathered}
\hat{\delta}=\frac{\bar{x}-\bar{y}}{\sqrt{s_{x}^{2}+s_{y}^{2}}} \\
\hat{f}=\left(s_{x}^{2}+s_{y}^{2}\right)^{2} /\left(\frac{s_{x}^{2}}{n-1}+\frac{s_{y}^{2}}{m-1}\right)
\end{gathered}
$$


and

$$
\hat{M}=\frac{s_{x}^{2}+s_{y}^{2}}{s_{x}^{2} / n+s_{y}^{2} / m}
$$

A $(1-\alpha)$ confidence lower bound can be obtained as $\Phi\left(\delta_{l}\right)$ with $\delta_{l}=\hat{\delta}-\left(\frac{1}{\hat{M}}+\frac{\hat{\delta}^{2}}{2 \hat{f}}\right) z_{1-\alpha}$. The simulations show that the coverage is not badly influenced by unequal sample sizes.

Church and Harris (1970) obtain confidence intervals for $P(Y<X)$ under the assumptions that $X$ and $Y$ are independently normally distributed and the distribution of $Y$ is known. Downton (1973) derives the minimum variance unbiased estimator of $P(Y<X)$ under the same assumptions. Approximations to this "best" estimator are obtained, and they are suggested as alternatives to the asymptotically equivalent estimator used by Church and Harris to obtain confidence intervals for that probability; they generalize the estimator to the case the distribution of $Y$ is unknown. Weerahandi and Johnson (1992) derive approximate confidence intervals for the reliability, based upon generalized $p$-values, when $X$ and $Y$ are independently normally distributed and all the parameters are unknown. Guo and Krishnamoorthy (2004) propose an approximate (one-sided) CI for $R$ by first computing a lower bound for $\delta$ and then transforming it to a lower bound for $R$ as done by Reiser and Guttman (1986). The proposal involves noncentral $t$ with a non-integer number of degrees of freedom and requires a numerical solution. More in detail, if we denote with $F(x ; d f, \xi)$ the noncentral $t$ cumulative distribution function with $d f$ degrees of freedom and noncentrality parameter $\xi$, a first lower bound $\delta_{1 L}$ is the solution of the equation

$$
F\left(\sqrt{\hat{m}_{1}} \hat{\delta} ; \hat{f}_{1}, \sqrt{\hat{m}_{1}} \delta_{1 L}\right)=1-\alpha
$$

where

$$
\hat{m}_{1}=\frac{n\left(1+\hat{q}_{1}\right)}{\hat{q}_{1}+n / m} ; \hat{f}_{1}=\frac{(n-1)\left(\hat{q}_{1}+1\right)^{2}}{\hat{q}_{1}^{2}+(n-1) /(m-1)} ; \hat{q}_{1}=s_{1}^{2} / s_{2}^{2}
$$

whereas a second lower bound $\delta_{2 L}$ is the solution of the equation

$$
F\left(\sqrt{\hat{m}_{2}} \hat{\delta} ; \hat{f}_{2}, \sqrt{\hat{m}_{2}} \delta_{2 L}\right)=1-\alpha
$$

where

$$
\hat{m}_{2}=\frac{m\left(1+\hat{q}_{2}\right)}{\hat{q}_{2}+m / n} ; \hat{f}_{1}=\frac{(m-1)\left(\hat{q}_{2}+1\right)^{2}}{\hat{q}_{2}^{2}+(m-1) /(n-1)} ; \hat{q}_{2}=s_{2}^{2} / s_{1}^{2}
$$

The actual lower bound for $R$ is obtained as

$$
\hat{R}_{L}=\Phi\left(\min \left\{\delta_{1 L}, \delta_{2 L}\right\}\right)
$$


Mukherjee and Mahiti (1998) develop an interval estimation procedure on the basis of the asymptotic normality of the maximum likelihood estimator (ML) of R and also provide interval estimation procedures based on variance stabilizing transformations such as logit and arcsine. Specifically, they consider the case of two independent Weibull distributions. For the same setup, Krishnamoorthy and Lin (2010) propose an alternative method using a generalized variable approach.

In the next Section, we will provide some new procedures to obtain an approximate CI for $R$.

\section{The proposals}

We will now describe our new method for the interval estimation of reliability, based upon two independent samples from $X$ and $Y$, denoted as $x$ and $y$, of size $n$ and $m$ respectively. The first is based upon parametric bootstrap, the second ones upon asymptotic results.

\subsection{A parametric bootstrap procedure}

Our first method is a straightforward extension and application of parametric bootstrap for the i.i.d. case (Efron and Tibshirani, 1994) to a two-sample case. It works as follows:

1. estimate the parameters of the r.v. $X$ and $Y$, through $\hat{\boldsymbol{\theta}}$ and $\hat{\boldsymbol{\lambda}}$ (e.g. maximum likelihood)

2. estimate $R$ as

$$
\hat{R}=\int_{-\infty}^{+\infty} f_{x}(x, \hat{\boldsymbol{\theta}}) \int_{-\infty}^{x} f_{y}(y, \hat{\boldsymbol{\lambda}}) d y d x
$$

For normal r.v. $R$ has an analytical expression depending upon the parameters of $X$ and $Y$ (see Equation (1)), and can be easily estimated e.g. through (see Downton, 1973)

$$
\hat{R}=\Phi\left(\frac{\bar{x}-\bar{y}}{\sqrt{s_{x}^{2}+s_{y}^{2}}}\right)
$$

or

$$
\hat{R}=\Phi\left(\frac{\bar{x}-\bar{y}}{\sqrt{\hat{\sigma}_{x}^{2}+\hat{\sigma}_{y}^{2}}}\right)
$$

\section{6}


which is the ML estimator of $R$

3. draw independently a bootstrap sample $x^{*}$ of size $n$ from a r.v. $X^{*}$ with the same distribution family of $X$ and parameter $\hat{\boldsymbol{\theta}}$ and a bootstrap sample $y^{*}$ of size $m$ from a r.v. $Y^{*}$ with the same distribution family of $Y$ and parameter $\hat{\lambda}$. In the normal case, $X^{*} \sim N\left(\bar{x}, \hat{\sigma}_{x}^{2}\right)$ (or $X^{*} \sim N\left(\bar{x}, s_{x}^{2}\right)$ ) and similarly for $Y^{*}$. Since all procedures depend on only the sample means and variances, for the well-known properties of the normal distribution, for this case we only have to generate $\bar{X}^{*} \sim N\left(\bar{x} ; s_{x}^{2} / n\right)$ and $S_{x}^{* 2} \sim s_{x}^{2} \chi_{n-1}^{2} /(n-1)$; similarly, $\bar{Y}^{*}$ and $S_{y}^{* 2}$ can be generated.

4. estimate $R^{*}$, using the same expression for the estimator used in 2 .

5. repeat steps 3. and 4. $B$ times ( $B$ sufficiently large, e.g. 2,000), thus obtaining the bootstrap distribution $\left\{\hat{R}^{*}\right\}$

6. estimate a $(1-\alpha)$ bootstrap percentile CI for $R$ from $\hat{R}^{*}$ distribution, taking the $\alpha / 2$ and $1-\alpha / 2$ quantiles:

$$
\left(\hat{R}_{\alpha / 2}^{*}, \hat{R}_{1-\alpha / 2}^{*}\right)
$$

or a $(1-\alpha)$ bootstrap percentile lower bound for $R$ as $\hat{R}_{\alpha}^{*}$

The procedure is quite easy, even if time-consuming, due to the bootstrap step which requires a huge number of runs $(2,000$ is a recommended value for percentile bootstrap). Here it is applied to the normal case; indeed, it only preferably requires that the reliability has got a known expression for the two distributions; otherwise the reliability estimate $\hat{R}$ has to be numerically computed, and the bootstrap estimates $\hat{R}^{*}$ too. It is a general purpose algorithm, since it can be applied to all possible distribution families; anyway it requires an a priori parametric hypothesis about the distribution of $X$ and $Y$, which is needed in order to estimate the parameters and the model reliability. The interval estimation is indeed performed only recalling the (parametric) bootstrap principle, which is independently applied to the two samples, with no concern about the different sample size, and then used to get a bootstrap distribution for $\hat{R}^{*}$, which is an estimate for the unknown distribution of $\hat{R}$.

Actually, only Guo and Krishnamoorthy (2004) have considered the bootstrap method for reliability estimation, specifically as a way to derive approximate test for reliability, at least for the normal case; yet, they have observed that the parametric bootstrap does not give satisfactory 
results: their extensive simulation studies showed that the test based on parametric bootstrap method is liberal, i.e. its size is far greater than the nominal level. We will see that the bootstrap method can provide acceptable results, even if not as good as other approximate methods; yet it turns out as an alternative approach when all the parameters are unknown.

\subsection{Confidence intervals based upon asymptotic results}

Another way to obtain approximate confidence intervals for reliability is to recall some asymptotic results and/or to use some variance stabilizing functions. Asymptotic confidence intervals can be easily obtained. The asymptotic variance of the ML estimator $\hat{R}$ can be derived following the same steps used in Mukherjee and Sharan (1985), and it can be estimated by

$$
v(\hat{R})=\frac{1}{2 \pi s^{2}} e^{-(\bar{x}-\bar{y})^{2} / s^{2}}\left[s_{x}^{2} / n+s_{y}^{2} / m+1 / 2 \frac{(\bar{x}-\bar{y})^{2}}{s^{4}}\left(s_{x}^{4} / n+s_{y}^{4} / m\right)\right]
$$

where $s^{2}=s_{x}^{2}+s_{y}^{2}$. A confidence interval for $R$ can be then built using the variance estimate above; however, for the same reason recalled by Reiser and Guttman, it may be better to adopt some specific modifications in order to produce better CI. For example, it is possible to find first the estimate of the asymptotic variance of the ML estimator of $\delta$,

$$
\hat{d}=\frac{\bar{x}-\bar{y}}{\hat{\sigma}_{x}^{2}+\hat{\sigma}_{y}^{2}}
$$

that can be obtained as:

$$
v(\hat{d})=\frac{1}{s^{2}}\left(s_{x}^{2} / n+s_{y}^{2} / m+\frac{1}{2} \frac{(\bar{x}-\bar{y})^{2}}{s^{4}}\left(s_{x}^{2} / n+s_{y}^{2} / m\right)\right)
$$

and can be employed to construct an approximate $(1-\alpha)$ CI for $d$ :

$$
\left(d_{L}, d_{U}\right)=\left(\hat{d} \pm z_{1-\alpha / 2} \sqrt{v(\hat{d})}\right)
$$

and then an approximate $(1-\alpha)$ CI for $R$ :

$$
\left(\Phi\left(d_{L}\right), \Phi\left(d_{U}\right)\right)
$$

or an approximate $(1-\alpha)$ lower bound as $\Phi\left(\hat{d}-z_{1-\alpha} \sqrt{v(\hat{d})}\right)$.

The variance estimate of $\hat{R}$ can be employed to build a CI for $R$ by previously "stabilizing" it through a proper transformation. For a normalizing transformation $g(\hat{R})$, the approximate 
variance can be obtained, according to the delta method, as

$$
V(g(\hat{R})) \simeq\left[g^{\prime}(R)\right]^{2} V(\hat{R})
$$

Then, see Krishnamoorthy and Lin (2010), for the logit transformation $g(R)=\ln \left(\frac{R}{1-R}\right)$ we get the approximate $(1-\alpha) \mathrm{CI}$

$$
\left(e^{L}\left(1+e^{L}\right)^{-1}, e^{U}\left(1+e^{U}\right)^{-1}\right)
$$

where $L$ and $U$ are the lower and upper bound of the CI:

$$
\left(\ln \left(\frac{\hat{R}}{1-\hat{R}}\right) \pm z_{1-\alpha / 2} \frac{\sqrt{v(\hat{R})}}{\hat{R}(1-\hat{R})}\right)
$$

For the arcsin transformation, the approximate $(1-\alpha) \mathrm{CI}$ is given by

$$
\left(\sin ^{2}(L), \sin ^{2}(U)\right)
$$

where $L$ and $U$ are the lower and upper bound of the CI:

$$
\left(\sin ^{-1}(\sqrt{\hat{R}}) \pm z_{1-\alpha / 2} \sqrt{\frac{v(\hat{R})}{4 \hat{R}(1-\hat{R})}}\right)
$$

Analogously, an approximate $(1-\alpha)$ lower bound can be obtained for $R$ recalling the logit and arcsin transformations. The three latest confidence intervals are based upon the asymptotic properties of the ML estimator, so we expect they may fail or show poor results when the sample sizes $n$ and $m$ are small. The simulation study we present in the next Section will point out if and when this expectation is well-founded.

\section{Simulation study}

The simulation study we performed aims at empirically checking the statistical properties of the proposed estimators and its competitors, specifically coverage and average length at a nominal level (95\%). The simulation study works as follows:

1. set the parameters for the r.v. $X$ and $Y$

2. compute $R$ 
3. draw independently a sample $x$ of size $n$ from $X$ and a sample $y$ of size $m$ from $Y$

4. estimate $R$ and a CI (either two-sided or one-sided) for $R$

5. check out if the CI computed at the previous step contains $R$; compute its length

6. repeat steps 3-5 nSim $(10,000)$ times and compute the overall CI coverage and average length

The confidence intervals empirically investigated by simulation are:

- Reiser and Guttman approximate CI (RG, Eq.3)

- Guo and Krishnamoorthy approximate CI (GK, Eq.4)

- bootstrap percentile CI (B, Eq.5)

- approximate CI based on asymptotic variance of $d$ (AS, Eq.6)

- approximate $\mathrm{CI}$ based on logit transformation (LO, Eq.7)

- approximate CI based on sin transformation (SIN, Eq.8)

We considered different scenarios, each corresponding to a different combination of distributional parameters (and thus different reliabilities), reported in Table 1, and sample sizes. Without any loss in generality, we fixed $Y \sim N\left(\mu_{y}=0, \sigma_{y}=1\right)$ and varied the parameters of $X \sim N\left(\mu_{x}, \sigma_{x}\right)$. We set the parameters in order to get a high value for the reliability, since, as we have stressed before, in real practice we usually look for high reliability for the study component/system.

\begin{tabular}{ccccccc}
\hline parameter & \multicolumn{7}{c}{ values } \\
\hline$\mu_{x}$ & 2 & 3 & 4 & 2 & 3 & 4 \\
$\mu_{y}$ & 0 & 0 & 0 & 0 & 0 & 0 \\
$\sigma_{x}$ & 1 & 1 & 1 & 3 & 3 & 3 \\
$\sigma_{y}$ & 1 & 1 & 1 & 1 & 1 & 1 \\
\hline$R$ & 0.9214 & 0.9831 & 0.9977 & 0.7365 & 0.8286 & 0.8970 \\
\hline
\end{tabular}

Table 1: Parameters for the simulation study

The Monte Carlo (MC) relative bias of $R$ has been taken under control by checking out the condition

$$
\left|\left(E_{M C}(\hat{R})-R\right) / R\right| \leq 2 \%
$$




\begin{tabular}{|c|c|c|c|c|c|c|c|c|c|c|c|}
\hline \multicolumn{2}{|c|}{$\mu_{x}=2$} & \multicolumn{2}{|c|}{$\mu_{x}=3$} & \multicolumn{2}{|c|}{$\mu_{x}=4$} & \multicolumn{2}{|c|}{$\mu_{x}=2$} & \multicolumn{2}{|c|}{$\mu_{x}=3$} & \multicolumn{2}{|c|}{$\mu_{x}=4$} \\
\hline$\sigma_{x}=1$ & $\sigma_{x}=3$ & $\sigma_{x}=1$ & $\sigma_{x}=3$ & $\sigma_{x}=1$ & $\sigma_{x}=3$ & $\sigma_{x}=1$ & $\sigma_{x}=3$ & $\sigma_{x}=1$ & $\sigma_{x}=3$ & $\sigma_{x}=1$ & $\sigma_{x}=3$ \\
\hline \multicolumn{6}{|c|}{$n=10, m=10$} & \multicolumn{6}{|c|}{$n=20, m=50$} \\
\hline+0.257 & +1.298 & -0.167 & +1.054 & -0.100 & +0.338 & +0.083 & +0.804 & -0.057 & +0.544 & -0.048 & +0.118 \\
\hline \multicolumn{6}{|c|}{$n=10, m=20$} & \multicolumn{6}{|c|}{$n=50, m=10$} \\
\hline+0.152 & +1.488 & -0.115 & +1.146 & +0.588 & +0.588 & +0.087 & +0.477 & -0.091 & +0.175 & -0.064 & +0.343 \\
\hline \multicolumn{6}{|c|}{$n=10, m=50$} & \multicolumn{6}{|c|}{$n=50, m=20$} \\
\hline+0.130 & +1.236 & -0.107 & +0.995 & -0.067 & +0.733 & +0.096 & +0.284 & -0.042 & +0.256 & -0.051 & +0.123 \\
\hline \multicolumn{6}{|c|}{$n=20, m=10$} & \multicolumn{6}{|c|}{$n=50, m=50$} \\
\hline+0.246 & +0.675 & -0.084 & +0.670 & -0.080 & +0.420 & +0.065 & +0.243 & -0.038 & +0.253 & -0.034 & +0.000 \\
\hline & & $n=20$ & $\imath=20$ & & & & & & & & \\
\hline+0.131 & +0.431 & -0.040 & +0.279 & -0.040 & +0.683 & & & & & & \\
\hline
\end{tabular}

Table 2: Simulation results: $\hat{R}$ percentage relative bias

Actually, the reliability estimator employed is not unbiased, and the MC relative error is an estimate of its relative bias $(\mathrm{E}(\hat{R})-R) / R$.

The results of the simulation study are reported in Tables 3 and 4 (coverage and average length of the two-sided CIs) and Tables 5 and 6 (coverage and average lower bound of onesided CIs). The MC percentage relative bias of $\hat{R}$ is reported in Table 2.

With regard to the behavior of $\hat{R}$, even if the ML estimator is only asymptotically unbiased, its MC bias is here shown to be negligible, since its absolute value is always smaller than $1.5 \%$. As we would have expected, sample sizes affect it significantly: for $n=m=50$ it decreases to $0.25 \%$. The $\mathrm{MC}$ bias is also strongly affected by the experimental conditions: in particular, it takes negative values when the true $R$ is closer to 1 , while takes the greatest positive values when $\mathrm{R}$ is nearer to 0.5 .

Looking at the results for two-sided CIs, it is clearly visible that Reiser and Guttman inferential procedure gives very good results, which seem not to be affected by the samples' sizes and by their discrepancy, even if one can note a slight departure of coverage rate from the nominal level when $n=10$ and $m=50$. The asymptotic normal CI provide very good results too, in terms of coverage, which is always very close to the nominal level, yet its performance is a bit worse than Reiser and Guttman's. The bootstrap proposal show on average a good performance, assuring a coverage always greater than $90 \%$ even for small samples; moreover, it is the method that provides, on average, the shortest confidence interval, under each experimental

11 


\begin{tabular}{|c|c|c|c|c|c|c|c|c|c|c|c|c|c|}
\hline \multirow[t]{2}{*}{ method } & \multicolumn{2}{|c|}{$\mu_{x}=2$} & \multicolumn{2}{|c|}{$\mu_{x}=3$} & \multicolumn{2}{|c|}{$\mu_{x}=4$} & \multirow[t]{2}{*}{ method } & \multicolumn{2}{|c|}{$\mu_{x}=2$} & \multicolumn{2}{|c|}{$\mu_{x}=3$} & \multicolumn{2}{|c|}{$\mu_{x}=4$} \\
\hline & $\sigma_{x}=1$ & $\sigma_{x}=3$ & $\sigma_{x}=1$ & $\sigma_{x}=3$ & $\sigma_{x}=1$ & $\sigma_{x}=3$ & & $\sigma_{x}=1$ & $\sigma_{x}=3$ & $\sigma_{x}=1$ & $\sigma_{x}=3$ & $\sigma_{x}=1$ & $\sigma_{x}=3$ \\
\hline$n=10, m=10$ & & & & & & & $n=20, m=50$ & & & & & & \\
\hline RG & 0.954 & 0.943 & 0.957 & 0.950 & 0.959 & 0.951 & RG & 0.952 & 0.949 & 0.951 & 0.945 & 0.957 & 0.949 \\
\hline B & 0.910 & 0.896 & 0.899 & 0.907 & 0.894 & 0.901 & B & 0.935 & 0.927 & 0.926 & 0.922 & 0.939 & 0.926 \\
\hline AS & 0.950 & 0.939 & 0.952 & 0.947 & 0.949 & 0.950 & AS & 0.951 & 0.948 & 0.948 & 0.942 & 0.956 & 0.948 \\
\hline LO & 0.978 & 0.973 & 0.983 & 0.970 & 0.994 & 0.983 & LO & 0.960 & 0.953 & 0.970 & 0.954 & 0.974 & 0.967 \\
\hline SIN & 0.922 & 0.911 & 0.906 & 0.923 & 0.887 & 0.922 & SIN & 0.936 & 0.935 & 0.920 & 0.927 & 0.926 & 0.938 \\
\hline$n=10, m=20$ & & & & & & & $n=50, m=10$ & & & & & & \\
\hline RG & 0.950 & 0.944 & 0.949 & 0.945 & 0.952 & 0.944 & RG & 0.938 & 0.951 & 0.940 & 0.950 & 0.942 & 0.949 \\
\hline B & 0.915 & 0.903 & 0.897 & 0.899 & 0.891 & 0.899 & B & 0.905 & 0.936 & 0.899 & 0.935 & 0.889 & 0.930 \\
\hline AS & 0.947 & 0.941 & 0.945 & 0.942 & 0.945 & 0.939 & AS & 0.935 & 0.950 & 0.935 & 0.949 & 0.932 & 0.948 \\
\hline LO & 0.977 & 0.963 & 0.973 & 0.960 & 0.988 & 0.978 & LO & 0.957 & 0.953 & 0.974 & 0.954 & 0.987 & 0.957 \\
\hline SIN & 0.923 & 0.919 & 0.918 & 0.915 & 0.874 & 0.909 & SIN & 0.911 & 0.941 & 0.894 & 0.938 & 0.869 & 0.933 \\
\hline$n=10, m=50$ & & & & & & & $n=50, m=20$ & & & & & & \\
\hline RG & 0.936 & 0.945 & 0.937 & 0.943 & 0.951 & 0.942 & RG & 0.952 & 0.945 & 0.949 & 0.948 & 0.959 & 0.953 \\
\hline B & 0.909 & 0.902 & 0.900 & 0.897 & 0.912 & 0.895 & B & 0.931 & 0.936 & 0.927 & 0.937 & 0.933 & 0.954 \\
\hline AS & 0.933 & 0.942 & 0.932 & 0.938 & 0.944 & 0.939 & AS & 0.950 & 0.946 & 0.947 & 0.948 & 0.956 & 0.951 \\
\hline LO & 0.953 & 0.958 & 0.972 & 0.968 & 0.988 & 0.975 & LO & 0.962 & 0.946 & 0.969 & 0.953 & 0.984 & 0.959 \\
\hline SIN & 0.915 & 0.919 & 0.896 & 0.913 & 0.891 & 0.912 & SIN & 0.932 & 0.938 & 0.919 & 0.940 & 0.916 & 0.951 \\
\hline$n=20, m=10$ & & & & & & $a^{2}$ & $n=50, m=50$ & & & & & & \\
\hline RG & 0.951 & 0.947 & 0.947 & 0.954 & 0.947 & 0.926 & $\mathrm{RG}$ & 0.953 & 0.951 & 0.948 & 0.950 & 0.951 & 0.936 \\
\hline B & 0.911 & 0.927 & 0.901 & 0.928 & 0.901 & 0.905 & B & 0.937 & 0.943 & 0.939 & 0.941 & 0.945 & 0.936 \\
\hline AS & 0.947 & 0.947 & 0.943 & 0.953 & 0.943 & 0.925 & AS & 0.952 & 0.951 & 0.947 & 0.949 & 0.951 & 0.936 \\
\hline LO & 0.972 & 0.952 & 0.983 & 0.965 & 0.990 & 0.961 & LO & 0.958 & 0.953 & 0.961 & 0.953 & 0.965 & 0.942 \\
\hline SIN & 0.919 & 0.932 & 0.900 & 0.936 & 0.881 & 0.907 & SIN & 0.939 & 0.946 & 0.935 & 0.944 & 0.937 & 0.939 \\
\hline$n=20, m=20$ & & & & & & & & & & & & & \\
\hline RG & 0.948 & 0.943 & 0.954 & 0.949 & 0.966 & 0.953 & & & & & & & \\
\hline B & 0.926 & 0.927 & 0.920 & 0.929 & 0.924 & 0.906 & & & & & & & \\
\hline AS & 0.945 & 0.942 & 0.950 & 0.948 & 0.961 & 0.949 & & & & & & & \\
\hline LO & 0.965 & 0.947 & 0.982 & 0.948 & 0.992 & 0.972 & & & & & & & \\
\hline SIN & 0.929 & 0.932 & 0.916 & 0.939 & 0.905 & 0.915 & & & & & & & \\
\hline
\end{tabular}

Table 3: Simulation results: CI coverage rate 


\begin{tabular}{|c|c|c|c|c|c|c|c|c|c|c|c|c|c|}
\hline \multirow[t]{2}{*}{ method } & \multicolumn{2}{|c|}{$\mu_{x}=2$} & \multicolumn{2}{|c|}{$\mu_{x}=3$} & \multicolumn{2}{|c|}{$\mu_{x}=4$} & \multirow{2}{*}{ method } & \multicolumn{2}{|c|}{$\mu_{x}=2$} & \multicolumn{2}{|c|}{$\mu_{x}=3$} & \multicolumn{2}{|c|}{$\mu_{x}=4$} \\
\hline & $\sigma_{x}=1$ & $\sigma_{x}=3$ & $\sigma_{x}=1$ & $\sigma_{x}=3$ & $\sigma_{x}=1$ & $\sigma_{x}=3$ & & $\sigma_{x}=1$ & $\sigma_{x}=3$ & $\sigma_{x}=1$ & $\sigma_{x}=3$ & $\sigma_{x}=1$ & $\sigma_{x}=3$ \\
\hline$n=10, m=10$ & & & & & & & $n=20, m=50$ & & & & & & \\
\hline RG & 0.243 & 0.402 & 0.123 & 0.349 & 0.052 & 0.292 & RG & 0.136 & 0.288 & 0.055 & 0.247 & 0.017 & 0.200 \\
\hline B & 0.198 & 0.398 & 0.080 & 0.317 & 0.026 & 0.240 & B & 0.125 & 0.287 & 0.046 & 0.236 & 0.012 & 0.181 \\
\hline AS & 0.238 & 0.399 & 0.118 & 0.344 & 0.048 & 0.285 & AS & 0.135 & 0.287 & 0.054 & 0.245 & 0.016 & 0.198 \\
\hline LO & 0.294 & 0.409 & 0.271 & 0.374 & 0.422 & 0.345 & LO & 0.142 & 0.289 & 0.063 & 0.252 & 0.023 & 0.211 \\
\hline SIN & 0.213 & 0.395 & 0.088 & 0.329 & 0.028 & 0.259 & SIN & 0.130 & 0.286 & 0.047 & 0.241 & 0.012 & 0.189 \\
\hline$n=10, m=20$ & & 2 & & & & & $n=50, m=10$ & & & & & & \\
\hline RG & 0.206 & 0.393 & 0.096 & 0.341 & 0.036 & 0.283 & RG & 0.181 & 0.222 & 0.081 & 0.186 & 0.029 & 0.144 \\
\hline B & 0.175 & 0.389 & 0.068 & 0.310 & 0.020 & 0.233 & B & 0.157 & 0.220 & 0.060 & 0.181 & 0.017 & 0.135 \\
\hline AS & 0.203 & 0.389 & 0.093 & 0.336 & 0.034 & 0.277 & AS & 0.178 & 0.221 & 0.079 & 0.186 & 0.027 & 0.143 \\
\hline LO & 0.230 & 0.398 & 0.144 & 0.361 & 0.129 & 0.328 & $\mathrm{LO}$ & 0.194 & 0.222 & 0.105 & 0.189 & 0.060 & 0.148 \\
\hline SIN & 0.186 & 0.387 & 0.072 & 0.323 & 0.020 & 0.252 & SIN & 0.167 & 0.221 & 0.063 & 0.184 & 0.017 & 0.139 \\
\hline$n=10, m=50$ & & & & & & & $n=50, m=20$ & & & & & & \\
\hline RG & 0.180 & 0.388 & 0.082 & 0.337 & 0.030 & 0.278 & RG & 0.136 & 0.204 & 0.055 & 0.172 & 0.017 & 0.135 \\
\hline B & 0.156 & 0.383 & 0.060 & 0.307 & 0.018 & 0.228 & B & 0.125 & 0.204 & 0.045 & 0.168 & 0.012 & 0.128 \\
\hline AS & 0.177 & 0.384 & 0.079 & 0.332 & 0.028 & 0.272 & AS & 0.135 & 0.204 & 0.054 & 0.172 & 0.016 & 0.134 \\
\hline $\mathrm{LO}$ & 0.193 & 0.392 & 0.105 & 0.356 & 0.061 & 0.318 & $\mathrm{LO}$ & 0.142 & 0.204 & 0.062 & 0.174 & 0.023 & 0.138 \\
\hline SIN & 0.166 & 0.382 & 0.064 & 0.320 & 0.018 & 0.248 & SIN & 0.129 & 0.204 & 0.047 & 0.170 & 0.012 & 0.131 \\
\hline$n=20, m=10$ & & & & & & & $n=50, m=50$ & & & & & & \\
\hline $\mathrm{RG}$ & 0.205 & 0.308 & 0.095 & 0.263 & 0.037 & 0.209 & RG & 0.102 & 0.192 & 0.038 & 0.163 & 0.010 & 0.129 \\
\hline B & 0.174 & 0.308 & 0.067 & 0.250 & 0.021 & 0.186 & B & 0.097 & 0.192 & 0.034 & 0.160 & 0.008 & 0.123 \\
\hline AS & 0.201 & 0.307 & 0.092 & 0.261 & 0.035 & 0.206 & AS & 0.101 & 0.192 & 0.038 & 0.162 & 0.010 & 0.128 \\
\hline LO & 0.229 & 0.310 & 0.143 & 0.270 & 0.131 & 0.223 & LO & 0.104 & 0.192 & 0.041 & 0.164 & 0.012 & 0.131 \\
\hline SIN & 0.185 & 0.307 & 0.071 & 0.256 & 0.021 & 0.195 & SIN & 0.099 & 0.192 & 0.035 & 0.161 & 0.008 & 0.126 \\
\hline$n=20, m=20$ & & & & & & & & & & & & & \\
\hline $\mathrm{RG}$ & 0.165 & 0.296 & 0.070 & 0.255 & 0.022 & 0.200 & & & & & & & \\
\hline B & 0.148 & 0.296 & 0.054 & 0.243 & 0.015 & 0.179 & & & & & & & \\
\hline AS & 0.163 & 0.295 & 0.068 & 0.253 & 0.022 & 0.198 & & & & & & & \\
\hline $\mathrm{LO}$ & 0.176 & 0.298 & 0.087 & 0.261 & 0.039 & 0.212 & & & & & & & \\
\hline SIN & 0.154 & 0.295 & 0.056 & 0.249 & 0.014 & 0.187 & & & & & & & \\
\hline
\end{tabular}

Table 4: Simulation results: CI average width 


\begin{tabular}{|c|c|c|c|c|c|c|c|c|c|c|c|c|c|}
\hline \multirow[t]{2}{*}{ method } & \multicolumn{2}{|c|}{$\mu_{x}=2$} & \multicolumn{2}{|c|}{$\mu_{x}=3$} & \multicolumn{2}{|c|}{$\mu_{x}=4$} & \multirow[t]{2}{*}{ method } & \multicolumn{2}{|c|}{$\mu_{x}=2$} & \multicolumn{2}{|c|}{$\mu_{x}=3$} & \multicolumn{2}{|c|}{$\mu_{x}=4$} \\
\hline & $\sigma_{x}=1$ & $\sigma_{x}=3$ & $\sigma_{x}=1$ & $\sigma_{x}=3$ & $\sigma_{x}=1$ & $\sigma_{x}=3$ & & $\sigma_{x}=1$ & $\sigma_{x}=3$ & $\sigma_{x}=1$ & $\sigma_{x}=3$ & $\sigma_{x}=1$ & $\sigma_{x}=3$ \\
\hline$n=10, m=10$ & & & & & & & $n=20, m=50$ & & & & & & \\
\hline RG & 0.958 & 0.949 & 0.958 & 0.943 & 0.951 & 0.951 & RG & 0.956 & 0.943 & 0.952 & 0.946 & 0.956 & 0.949 \\
\hline GK & 0.964 & 0.952 & 0.964 & 0.950 & 0.958 & 0.957 & GK & 0.959 & 0.947 & 0.956 & 0.952 & 0.959 & 0.950 \\
\hline B & 0.887 & 0.891 & 0.863 & 0.874 & 0.851 & 0.850 & B & 0.919 & 0.914 & 0.924 & 0.898 & 0.920 & 0.889 \\
\hline AS & 0.952 & 0.945 & 0.953 & 0.938 & 0.941 & 0.947 & AS & 0.953 & 0.942 & 0.950 & 0.943 & 0.950 & 0.948 \\
\hline LO & 1.000 & 0.960 & 1.000 & 0.984 & 1.000 & 1.000 & LO & 0.962 & 0.945 & 0.970 & 0.955 & 0.981 & 0.958 \\
\hline SIN & 0.906 & 0.908 & 0.883 & 0.895 & 0.860 & 0.889 & SIN & 0.922 & 0.926 & 0.919 & 0.917 & 0.904 & 0.901 \\
\hline$n=10, m=20$ & & & & & & & $n=50, m=10$ & & & & & & \\
\hline RG & 0.947 & 0.940 & 0.945 & 0.936 & 0.940 & 0.939 & RG & 0.926 & 0.950 & 0.926 & 0.950 & 0.917 & 0.950 \\
\hline GK & 0.952 & 0.944 & 0.953 & 0.941 & 0.948 & 0.949 & GK & 0.946 & 0.960 & 0.939 & 0.955 & 0.929 & 0.957 \\
\hline B & 0.883 & 0.879 & 0.858 & 0.872 & 0.850 & 0.865 & B & 0.876 & 0.926 & 0.855 & 0.917 & 0.848 & 0.917 \\
\hline AS & 0.942 & 0.934 & 0.942 & 0.930 & 0.936 & 0.932 & AS & 0.923 & 0.950 & 0.922 & 0.948 & 0.914 & 0.950 \\
\hline LO & 0.977 & 0.954 & 1.000 & 0.975 & 1.000 & 0.992 & LO & 0.946 & 0.951 & 0.965 & 0.954 & 0.989 & 0.960 \\
\hline SIN & 0.894 & 0.906 & 0.869 & 0.896 & 0.848 & 0.887 & SIN & 0.885 & 0.935 & 0.856 & 0.923 & 0.83 & 0.923 \\
\hline$n=10, m=50$ & & & & & & & $n=50, m=20$ & & & & & & \\
\hline RG & 0.944 & 0.947 & 0.941 & 0.941 & 0.938 & 0.939 & RG & 0.950 & 0.954 & 0.948 & 0.955 & 0.949 & 0.954 \\
\hline GK & 0.953 & 0.951 & 0.952 & 0.950 & 0.955 & 0.952 & GK & 0.953 & 0.959 & 0.952 & 0.958 & 0.953 & 0.959 \\
\hline B & 0.890 & 0.889 & 0.879 & 0.874 & 0.864 & 0.859 & B & 0.912 & 0.923 & 0.900 & 0.916 & 0.895 & 0.913 \\
\hline AS & 0.939 & 0.945 & 0.939 & 0.939 & 0.930 & 0.932 & AS & 0.949 & 0.954 & 0.946 & 0.955 & 0.948 & 0.953 \\
\hline LO & 0.956 & 0.953 & 0.970 & 0.966 & 0.987 & 0.984 & LO & 0.956 & 0.954 & 0.974 & 0.958 & 0.983 & 0.961 \\
\hline SIN & 0.901 & 0.913 & 0.879 & 0.897 & 0.850 & 0.889 & SIN & 0.917 & 0.934 & 0.891 & 0.926 & 0.872 & 0.921 \\
\hline$n=20, m=10$ & & & & & & & $n=50, m=50$ & & & & & & \\
\hline RG & 0.942 & 0.949 & 0.943 & 0.948 & 0.946 & 0.945 & RG & 0.962 & 0.956 & 0.957 & 0.957 & 0.954 & 0.953 \\
\hline GK & 0.952 & 0.951 & 0.952 & 0.952 & 0.959 & 0.948 & GK & 0.962 & 0.959 & 0.960 & 0.959 & 0.957 & 0.956 \\
\hline B & 0.875 & 0.908 & 0.857 & 0.894 & 0.856 & 0.875 & B & 0.940 & 0.939 & 0.937 & 0.927 & 0.932 & 0.923 \\
\hline AS & 0.934 & 0.947 & 0.937 & 0.946 & 0.941 & 0.943 & AS & 0.961 & 0.956 & 0.956 & 0.956 & 0.954 & 0.950 \\
\hline LO & 0.980 & 0.951 & 1.000 & 0.958 & 1.000 & 0.968 & LO & 0.965 & 0.956 & 0.966 & 0.957 & 0.976 & 0.957 \\
\hline SIN & 0.891 & 0.923 & 0.864 & 0.912 & 0.848 & 0.899 & SIN & 0.941 & 0.944 & 0.924 & 0.935 & 0.913 & 0.929 \\
\hline$n=20, m=20$ & & & & & & & & & & & & & \\
\hline RG & 0.948 & 0.932 & 0.950 & 0.933 & 0.953 & 0.934 & & & & & & & \\
\hline GK & 0.950 & 0.935 & 0.955 & 0.939 & 0.954 & 0.936 & & & & & & & \\
\hline B & 0.888 & 0.896 & 0.889 & 0.877 & 0.886 & 0.869 & & & & & & & \\
\hline AS & 0.947 & 0.931 & 0.948 & 0.926 & 0.949 & 0.930 & & & & & & & \\
\hline LO & 0.962 & 0.935 & 0.984 & 0.948 & 1.000 & 0.953 & & & & & & & \\
\hline SIN & 0.897 & 0.908 & 0.893 & 0.893 & 0.871 & 0.883 & & & & & & & \\
\hline
\end{tabular}

Table 5: Simulation results: one-sided CI coverage rate 


\begin{tabular}{|c|c|c|c|c|c|c|c|c|c|c|c|c|c|}
\hline \multirow[t]{2}{*}{ method } & \multicolumn{2}{|c|}{$\mu_{x}=2$} & \multicolumn{2}{|c|}{$\mu_{x}=3$} & \multicolumn{2}{|c|}{$\mu_{x}=4$} & \multirow[t]{2}{*}{ method } & \multicolumn{2}{|c|}{$\mu_{x}=2$} & \multicolumn{2}{|c|}{$\mu_{x}=3$} & \multicolumn{2}{|c|}{$\mu_{x}=4$} \\
\hline & $\sigma_{x}=1$ & $\sigma_{x}=3$ & $\sigma_{x}=1$ & $\sigma_{x}=3$ & $\sigma_{x}=1$ & $\sigma_{x}=3$ & & $\sigma_{x}=1$ & $\sigma_{x}=3$ & $\sigma_{x}=1$ & $\sigma_{x}=3$ & $\sigma_{x}=1$ & $\sigma_{x}=3$ \\
\hline$n=10, m=10$ & & & & & & & $n=20, m=50$ & & & & & & \\
\hline RG & 0.775 & 0.535 & 0.902 & 0.637 & 0.963 & 0.725 & RG & 0.845 & 0.597 & 0.948 & 0.701 & 0.987 & 0.787 \\
\hline GK & 0.769 & 0.530 & 0.897 & 0.631 & 0.960 & 0.717 & GK & 0.842 & 0.594 & 0.947 & 0.697 & 0.986 & 0.784 \\
\hline B & 0.817 & 0.565 & 0.934 & 0.681 & 0.980 & 0.776 & B & 0.858 & 0.612 & 0.956 & 0.723 & 0.990 & 0.811 \\
\hline AS & 0.778 & 0.537 & 0.905 & 0.641 & 0.965 & 0.729 & AS & 0.845 & 0.597 & 0.949 & 0.702 & 0.987 & 0.788 \\
\hline LO & 0.749 & 0.533 & 0.827 & 0.625 & 0.746 & 0.696 & LO & 0.843 & 0.597 & 0.945 & 0.699 & 0.984 & 0.782 \\
\hline SIN & 0.806 & 0.556 & 0.927 & 0.667 & 0.977 & 0.759 & SIN & 0.856 & 0.607 & 0.956 & 0.715 & 0.990 & 0.804 \\
\hline$n=10, m=20$ & & & & & & & $n=50, m=10$ & & & & & & \\
\hline RG & 0.802 & 0.542 & 0.921 & 0.645 & 0.973 & 0.732 & RG & 0.820 & 0.634 & 0.932 & 0.739 & 0.978 & 0.823 \\
\hline GK & 0.795 & 0.536 & 0.916 & 0.637 & 0.971 & 0.724 & GK & 0.811 & 0.630 & 0.926 & 0.735 & 0.975 & 0.820 \\
\hline B & 0.833 & 0.571 & 0.943 & 0.689 & 0.984 & 0.783 & B & 0.844 & 0.644 & 0.949 & 0.750 & 0.986 & 0.835 \\
\hline AS & 0.804 & 0.544 & 0.923 & 0.648 & 0.975 & 0.737 & AS & 0.822 & 0.635 & 0.933 & 0.739 & 0.979 & 0.823 \\
\hline LO & 0.790 & 0.540 & 0.896 & 0.635 & 0.933 & 0.709 & LO & 0.814 & 0.635 & 0.920 & 0.738 & 0.964 & 0.822 \\
\hline SIN & 0.826 & 0.562 & 0.939 & 0.673 & 0.983 & 0.766 & SIN & 0.839 & 0.640 & 0.946 & 0.747 & 0.986 & 0.832 \\
\hline$n=10, m=50$ & & & & & & & $n=50, m=20$ & & & & & & \\
\hline RG & 0.820 & 0.547 & 0.932 & 0.650 & 0.978 & 0.737 & RG & 0.847 & 0.643 & 0.949 & 0.746 & 0.987 & 0.828 \\
\hline GK & 0.811 & 0.540 & 0.926 & 0.641 & 0.975 & 0.728 & GK & 0.844 & 0.642 & 0.947 & 0.745 & 0.986 & 0.827 \\
\hline B & 0.844 & 0.576 & 0.949 & 0.693 & 0.986 & 0.786 & B & 0.860 & 0.651 & 0.957 & 0.756 & 0.990 & 0.839 \\
\hline AS & 0.822 & 0.549 & 0.934 & 0.653 & 0.979 & 0.741 & AS & 0.847 & 0.644 & 0.949 & 0.746 & 0.987 & 0.829 \\
\hline LO & 0.814 & 0.546 & 0.920 & 0.641 & 0.964 & 0.716 & LO & 0.845 & 0.644 & 0.945 & 0.746 & 0.984 & 0.827 \\
\hline SIN & 0.839 & 0.567 & 0.946 & 0.678 & 0.986 & 0.770 & SIN & 0.857 & 0.649 & 0.956 & 0.753 & 0.990 & 0.836 \\
\hline$n=20, m=10$ & & & & & & & $n=50, m=50$ & & & & & & \\
\hline RG & 0.804 & 0.592 & 0.922 & 0.697 & 0.974 & 0.784 & $\mathrm{RG}$ & 0.867 & 0.647 & 0.960 & 0.749 & 0.991 & 0.831 \\
\hline GK & 0.796 & 0.589 & 0.917 & 0.693 & 0.971 & 0.781 & GK & 0.867 & 0.647 & 0.960 & 0.748 & 0.991 & 0.830 \\
\hline B & 0.834 & 0.610 & 0.944 & 0.721 & 0.985 & 0.810 & B & 0.874 & 0.654 & 0.964 & 0.758 & 0.993 & 0.841 \\
\hline AS & 0.806 & 0.593 & 0.924 & 0.698 & 0.975 & 0.785 & AS & 0.867 & 0.648 & 0.960 & 0.750 & 0.991 & 0.831 \\
\hline LO & 0.792 & 0.592 & 0.924 & 0.694 & 0.933 & 0.777 & LO & 0.867 & 0.648 & 0.959 & 0.749 & 0.990 & 0.830 \\
\hline SIN & 0.827 & 0.604 & 0.940 & 0.713 & 0.984 & 0.803 & SIN & 0.873 & 0.652 & 0.964 & 0.755 & 0.993 & 0.838 \\
\hline$n=20, m=20$ & & & & & & & & & & & & & \\
\hline RG & 0.831 & 0.602 & 0.940 & 0.705 & 0.983 & 0.791 & & & & & & & \\
\hline GK & 0.829 & 0.599 & 0.939 & 0.702 & 0.982 & 0.788 & & & & & & & \\
\hline B & 0.850 & 0.618 & 0.952 & 0.728 & 0.988 & 0.816 & & & & & & & \\
\hline AS & 0.832 & 0.602 & 0.941 & 0.706 & 0.983 & 0.792 & & & & & & & \\
\hline LO & 0.826 & 0.602 & 0.931 & 0.703 & 0.975 & 0.786 & & & & & & & \\
\hline SIN & 0.847 & 0.613 & 0.951 & 0.721 & 0.988 & 0.809 & & & & & & & \\
\hline
\end{tabular}

Table 6: Simulation results: one-sided CI average lower bound 
condition. The little departure of coverage from the nominal level is a known issue of bootstrap interval estimation, see Schreuder and Williams (2000). The CI based on logit transformation gives always coverages greater than the nominal level, especially for high values of $R$; on the other hand, its average width is the largest. The CI based on sin transformation almost always give coverages never greater than 95\%, even for the largest samples' sizes; its performance in terms of both coverage and average width looks surprisingly similar to the bootstrap interval estimator. It is worthwhile noting that the two approximate methods proposed (LO and SIN), based on asymptotic results, perform well even for small sample sizes. It is also interesting to observe that for sample sizes moderately large $(n=m=50$, depending upon the other parameters) the performances of the different confidence intervals tend to get closer, in terms of both coverage $(\simeq 95 \%)$ and average length.

From the simulation study, it is interesting to note if and how the value of $R$ and the sample sizes $n$ and $m$ affect the performance of the interval estimators. With regard to the coverage, as already said, the samples' sizes and their discrepancy seem not to heavily affect the results (but, as expected, the overall best results are for $n=m=50$ ); the same can be told about the experimental conditions: the values of $\mu_{x}$ and $\sigma_{x}$ seem not to systematically affect the performances of the compared procedures. Even in the "worst" scenario $\left(\mu_{x}=4, \sigma_{x}=1\right.$, with $R$ very close to 1$)$ the methods keep working satisfactorily. Yet LO, for some scenarios, namely $\mu_{x}=4$ and $\sigma_{x}=1$, provides unreliable results. With regard to the average width, this is clearly influenced by the sample sizes and experimental conditions. The results get better (i.e. the CI are more precise) as the sample sizes increase and the reliability $R$ gets closer to 1 .

From the simulation results for one-sided confidence intervals, the overall superiority of GK procedure emerges, followed closed behind by RG and LO. The LO intervals here, in fact, allows almost always coverages greater than the nominal level, even if in some cases they are too conservative, providing also smaller lower bounds. The lower bounds based on asymptotic results provide satisfactory results, which only partially suffer from the finiteness of sample sizes. The bootstrap procedure gives worse results than in the two-sided cases: it attains coverage rates close to the nominal level only for large sample sizes, whereas they are often smaller than $90 \%$; it tends to give higher lower bounds than its competitors. Its "crude" use is not suggested; some proper modifications should be made, but the practitioner should 
also take into account the additional computation time this already time consuming method could bring. With regard to the SIN procedure, its performance is not totally satisfactory and reliable. One should also note an overall light worsening of the performance of asymptotic procedures passing from two-sided to one-sided CIs: this may be ascribed to the fact that asymptotic CIs overcover on one-tail and undercover on the other, and so overall coverage probabilities of asymptotic two-sided CIs turn out to be close to the nominal level $1-\alpha$. This behavior has been detected by Krishnamoorthy and Lin (2010) for the Weibull case.

\section{An example of application}

Table 7, taken from Duncan (1986), gives the results of measuring shear strength for spot welds for two different gauges of steel. Although not a stress-strength situation in the sense discussed in the introduction, this problem does fit our formulation, and we use it to show how the inferential procedures work on a practical case. Denoting with $X$ and $Y$ the r.v. modeling the strength of the two gauges of steel, the estimates computed on the data are $\bar{x}=975, \bar{y}=$ 480, $\hat{\sigma}_{x}^{2}=33055$ and $\hat{\sigma}_{y}^{2}=7345$. Assuming $X$ and $Y$ are independently normally distributed, the maximum likelihood estimator $\hat{R}$ has value 0.9931 . The confidence intervals calculated according each of the methods described in the previous sections are reported in Table 8 , in Table 9 the one-sided confidence intervals; they show that the proposed bootstrap methods provides the narrowest interval, confirming what we have already seen in the simulation study, while the LO method provides the largest one. RG, GK and AS bounds are very close to each other.

\section{Conclusions}

In this paper we propose a bootstrap approach and some approximate methods for the interval estimation of reliability in a stress-strength model, where both stress and strength are normally distributed with unknown parameters. We empirically investigate the performance of these methods and other existing ones, through a simulation study comprising different artificial scenarios. The results show the superiority (in particular when building one-sided tolerance 


\begin{tabular}{rr}
\hline$Y(m=10)$ & $X(n=10)$ \\
\hline 350 & 680 \\
380 & 800 \\
385 & 780 \\
450 & 885 \\
465 & 975 \\
485 & 1,025 \\
535 & 1,100 \\
555 & 1,030 \\
590 & 1,175 \\
605 & 1,300 \\
\hline
\end{tabular}

Table 7: Data on shear strengths of two gauges of steel

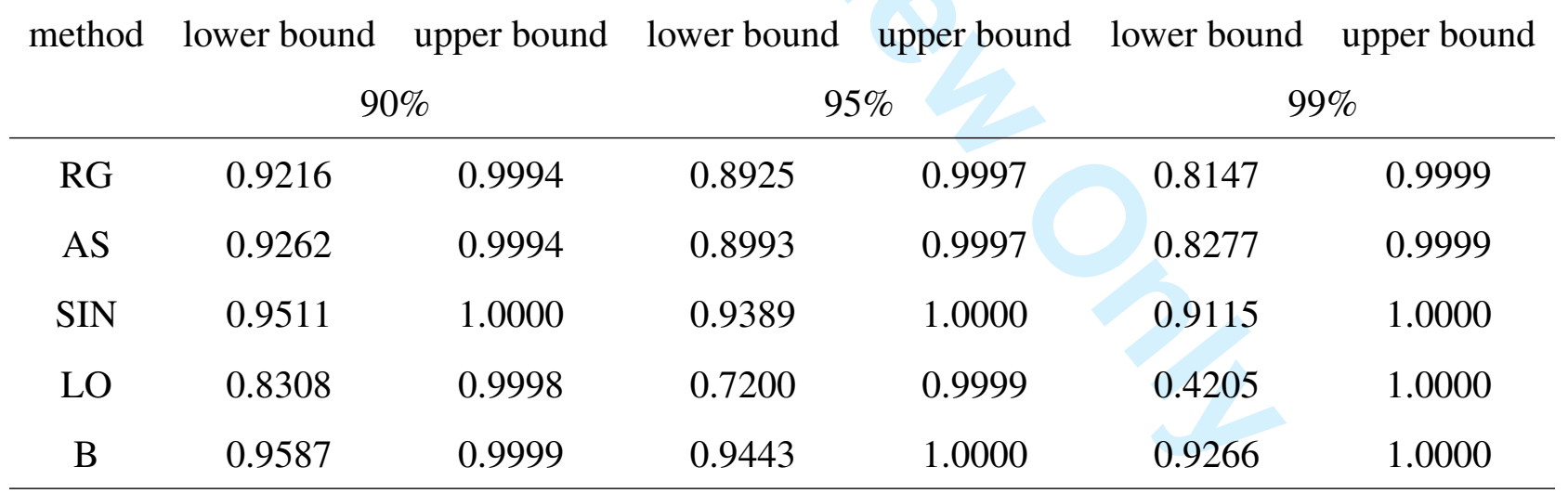

Table 8: Results for reliability on Duncan data: 90, 95, and 99\% confidence intervals. 


\begin{tabular}{cccc}
\hline method & $90 \%$ & $95 \%$ & $99 \%$ \\
\hline RG & 0.9473 & 0.9216 & 0.8496 \\
GK & 0.9416 & 0.9154 & 0.8467 \\
AS & 0.9499 & 0.9262 & 0.8599 \\
SIN & 0.9636 & 0.9511 & 0.9232 \\
LO & 0.9120 & 0.8308 & 0.5478 \\
B & 0.9685 & 0.9569 & 0.9303
\end{tabular}

Table 9: 90, 95, and 99\% lower bounds for reliability on Duncan data.

bounds) of two existing methods specifically conceived for the normal set-up. Our bootstrap proposal performs slightly worse than the other approximate methods in terms of coverage, but provides narrower intervals. Yet, the advantage of the bootstrap method versus its competitors lies in the actual computation of $\mathrm{CI}$, which does not require further formulas or approximations, but simply reuses the formula employed for point estimation. With regard to the estimators based on asymptotic results, their performance has been here satisfactory even for moderate sample sizes, however, a particular attention should be devoted if extending these results to other parametric families. The new bootstrap proposal can be easily extended, especially to the other family distributions for which an expression for the reliability $R$ is analytically derivable; it can be used in the independent set-up, as described here, but also generalized - with proper modifications - to the dependent set-up: future research shall then investigate the performance of the bootstrap interval estimator towards the existing ones for other parametric monovariate and bivariate families.

\section{Acknowledgments}

I would like to thank the referees for their helpful comments and suggestions.

I wish to dedicate this paper to the cherished memory of my mother and to my father. 


\section{References}

Birnbaum, Z. W. (1956). On a use of the Mann-Whitney statistic. Proceedings of the Third Berkeley Symposium on Mathematical Statistics and Probability, Vol. 1, Univ. of Calif. Press, 13-17.

Birnbaum, Z. W., McCarty, R. C. (1958). A distribution-free upper confidence bound for $P(Y<$ $X$ ) based on independent samples of $X$ and $Y$. Annals of Mathematical Statistics 29: 558562.

Chang D. S. (1995) Reliability bounds for the stress-strength model. Computers \& Industrial Engineering 29(1): 15-19

Church, J.D., Harris, B. (1970). The Estimation of Reliability from Stress-Strength Relationships. Technometrics 12(1): 551-558

Downton, F. (1973). The Estimation of $\operatorname{Pr}(Y<X)$ in the Normal Case. Technometrics 15(3): $551-558$

Efron, B., Tibshirani, R. (1994) An Introduction to the Bootstrap. Chapman \& Hall/CRC

Duncan, A. G. (1986). Quality and Industrial Statistics (5th ed.). Irwin, Homewood, IL

Guo, H., Krishnamoorthy, K. (2004). New Approximate Inferential Methods for the Reliability Parameter in a Stress-Strength Model: The Normal Case. Communications in Statistics Theory and Methods 33(7): 1715-1731

Hall, I.J. (1984). Approximate one-sided tolerance limits for the difference or sum of two independent normal variates. Journal of Quality Technology 16: 15-19

Harris, B., Soms, A. P. (1991) The Theory of Optimal Confidence Limits for Systems Reliability with Counterexamples for Results on Optimal Confidence Limits for Series Systems. Statistics \& Probability Letters 11(5): 411-417

Kotz, S., Lumelskii, Y., Pensky, M. (2003) The stress-strength model and its generalizations Theory and Applications. World Scientific Publishing 
Krishnamoorthy, K., Lin, Y. (2010). Confidence Limits for Stress-Strength Reliability Involving Weibull Models. Journal of Statistical Planning and Inference 140(7): 1754-1764

Mukherjee, S. P., Maiti, S. S. (1998). Stress-strength reliability in the Weibull case. In: Frontiers In Reliability 4: 231-248. World Scientific

Mukherjee, S.P., Sharan, L.K. (1985). Estimation of failure probability from a bivariate normal stress-strength distribution. Microelectronics Reliability 25: 699-702

Nadarajah, S., Kotz, S. (2005). Reliability For A Bivariate Gamma Distribution. Economic Quality Control 20(1): 111-119

Reiser, B., Guttman, I. (1986). Statistical Inference for $\operatorname{Pr}(Y<X)$ : The Normal Case. Technometrics, 28(3): 253-257

Roy, D. (1993) Estimation of failure probability under a bivariate normal stress-strength distribution. Microelectronics Reliability 33(15): 2285-2287

Schreuder, H. T., Williams, M.S. (2000). Reliability of Confidence Intervals Calculated by Bootstrap and Classical Methods Using the FIA 1-ha Plot Design. General Technical Report RMRSGTR-57. Fort Collins, U.S. Department of Agriculture, Forest Service, Rocky Mountain Research Station.

Weerahandi, S., Johnson, R. A. (1992). Testing Reliability in a Stress-Strength Model When $X$ and $Y$ Are Normally Distributed. Technometrics 34(1): 83-91 